\title{
Forecasts of Geomagnetic Activity in Canada by Linear Prediction Filtering
}

\author{
Hing-Lan LAM \\ Geophysics Division, Geological Survey of Canada, Department of Energy, Mines and Resources, \\ Ottawa, Ontario, Canada, KIA $0 Y 3$
}

(Received February 2, 1987)

\begin{abstract}
Forecasting of geomagnetic activity can be performed on the basis of the knowledge and experience of particular forecasters. However, it is more desirable to develop computer algorithms based on numerical techniques to perform the forecasting so that subjective judgement of humans can be reduced. The present study utilizes the ground geomagnetic data for the forecasts of geomagnetic activity. A linear prediction filtering technique is employed whereby the past values are linearly combined to predict future values based on Wiener linear prediction theory. Magnetic data from observatories in the polar cap, auroral zone and subauroral zone in Canada are used as input to the filter. While sporadic activities are unpredictable in this technique, the recurrent activities are well delineated by this method. The forecasts compare favourably with the actual data for the studied period which was at a time of solar minimum. It is shown by means of several verification schemes that linear prediction is a useful technique in objective and quantitative geomagnetic forecasting. The method is implemented into the new three-zone (i.e. polar cap, auroral and subauroral zones) forecasts of geomagnetic activity in Canada.
\end{abstract}

\section{Introduction}

Geomagnetic disturbances can cause serious interference on man-made systems and human activities. The electric currents that the disturbances induce in power lines, pipelines and telephone cables can lead to the degradation and reduced performance of such systems. In addition, radio communications, geophysical prospecting and defense surveillance are all greatly affected during geomagnetically active periods. Forecasts of geomagnetic activity are, therefore, needed for such diverse operations as national defense, aeromagnetic surveying, hydro power transmission and amateur radio operations by individuals.

The Department of Energy, Mines and Resources of the Government of Canada in Ottawa has been issuing forecasts of geomagnetic activity since 1974 (HRUSKA, 1979), and the prediction schemes that have been used up to the present are largely qualitative and empirical. As there is a developing need for increasingly reliable and ongoing forecasts it is now desirable to attempt to put the forecasting process on an

Geological Survey of Canada Contribution No. 41086. 
objective basis using all appropriate input data and algorithms that attempt to codify what has been a judgemental and successful procedures. To this end, a prediction filter based on Wiener linear prediction theory (WIENER, 1949) has been developed for medium term (27 days) forecasts of geomagnetic activity in Canada. The filter has been tested using data from Canadian Magnetic Observatories (for details regarding the observatories, see JANSEN VAN BEEK et al., 1986) distributed in the polar cap, auroral and subauroral zones which have magnetic characters distinct from each other. This paper presents results using such a prediction scheme and shows that it can be used to produce objective and quantitative forecasts.

\section{The Linear Prediction Filter}

The technique used is based on the least-squares criterion and it leads to filters which are linear. The mathematical theory of such filters was originally developed by WIENER (1949). The Wiener method of smoothing and prediction was adapted by LEVINSON (1949) to the case of finite-length discrete operators. The Wiener-Levinson algorithm was subsequently extended and implemented for digital use by Robinson (see for example, ROBINSON and TREITEL, 1967).

Briefly, the filter is determined by requiring that the sum of the mean-squareerrors between the desired outputs $z$ and the actual outputs $y$

$$
I=E\left\{\left(z_{\mathrm{t}}-y_{\mathrm{t}}\right)^{2}\right\}
$$

be minimized. Here, the desired output is the input advanced by a positive time increment and the actual output is the convolution of the input $x$ with the filter $f$

$$
y_{\mathrm{t}}=\sum_{s=0}^{m} f_{s} x_{\mathrm{t}-s}
$$

Substituting (2) into (1) and setting the partial derivative of $I$ with respect to each filter coefficient $f_{s}$ equal to zero to minimize $I$, a system of $m+1$ linear simultaneous equations, known as the normal equations, in the unknown filter coefficient $f_{s}$ can be obtained

$$
\sum_{s=0}^{m} f_{s} \theta_{x x}(j-s)=\theta_{z x}(j) \text { for } j=0,1,2, \ldots, m
$$

where $\theta_{x x}$ and $\theta_{z x}$ are known quantities of autocorrelations and cross correlations respectively. The solution of these equations yields the coefficients of the filter. Because the matrix of this set of equations is in the form of a Toeplitz matrix (i.e. a matrix with equal elements on any diagonal), a computationally efficient recursive technique developed by LEVINSON (1949) is used to solve the equations. A convolution of the filter with the input data yields the forecasts based on unit prediction distance. The filter coefficients are re-computed for each new set of data so 
that the resulting filter is data-adaptive and the relevant recurrent features of geomagnetic activity are utilized.

\section{Results}

The digital data used in this study were derived from the Canadian Magnetic Observatory Network. The daily means of the hourly ranges in the $X$ component of the earth's magnetic field (also referred to as the $D R X$ index for brevity) recorded in stations in Ottawa $\left(57.0^{\circ} \mathrm{N}\right.$ and $351.5^{\circ} \mathrm{E}$ measured in geomagnetic coordinates) in the subauroral zone, Fort Churchill $\left(67.8^{\circ} \mathrm{N}\right.$ and $\left.323.0^{\circ} \mathrm{E}\right)$ in the auroral zone and Resolute Bay $\left(83.1^{\circ} \mathrm{N}\right.$ and $\left.287.7^{\circ} \mathrm{E}\right)$ in the polar cap were used. The data covered a period of approximately one and a half years from April 24, 1984 to December 8, 1985 corresponding to the period of Bartels Solar Rotations 2060 to 2081. This period corresponds to a solar minimum when the recurrent tendency of geomagnetic activity is strong.

To determine the appropriate length of filter, varying lengths of filter with a fixed length of input data for the forecasts of the next 27 days (corresponding to 1 Bartel solar rotation) were tested. Here, the input data and the forecast values refer to the $D R X$ indices. It was found that features for the 27 days forecast began to stabilize starting at filter with length of 70 . There is not much improvement by using longer lengths of filter. This can also be justified by computing a parameter known as the normalized mean-square-error $Q_{0}$ as a function of the lengths of the filter.

The normalized mean-square-error $Q_{0}$ is defined as

$$
Q_{0}=I_{0} / \theta_{z z}(0)
$$

where

$$
I_{0}=\theta_{z z}(0)-\sum_{s=0}^{m} f_{s} \theta_{z x}(s)
$$

(RoBINSON and TREITEL, 1967). It is apparent that $Q_{0}$ lies between zero and one and that the smaller the value of $Q_{0}$, the better the prediction, with $Q_{0}=0$ corresponding to perfect prediction. A typical variation of $Q_{0}$ as a function of filter length in days is shown in Fig. 1. It can be seen that the curve levels out after a length of 50 and further increase in the length of the filter would not offer much improvement in the prediction.

Varying lengths of input data were also tested for the 27 days forecasts. It was found that using input data of more than 8 to 11 Bartels solar rotations did not produce noticeable changes in the forecast values. Also, results from using more rotations tend to reflect activities that occurred earlier. This may not be a desirable feature because the earlier activity may have subsided not to recur. It appears that 8 solar rotations of data with a filter length of one third of that is appropriate. This also corresponds to the life of coronal holes, the long-lived features on the sun, that in 


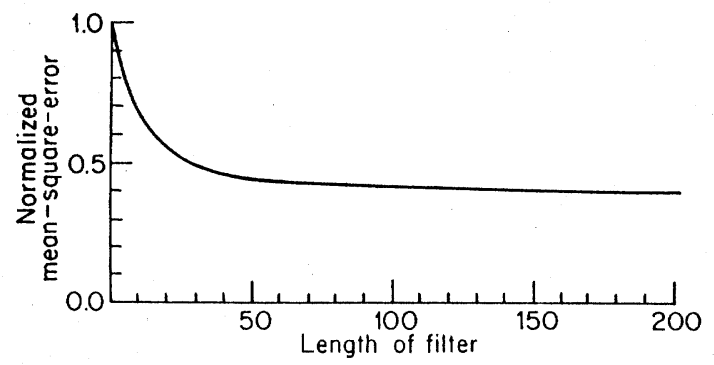

Fig. 1. Plot of normalized mean-square-error vs length of filter (days).

general last no more than 8 or 9 solar rotations. It is the high-speed streams emanating from coronal holes that predominantly cause the recurrent high geomagnetic activity.

Figures 2(a) and 2(b) show the forecast values (dotted line) and the actual data (solid line) from November 26, 1984 to December 8, 1985 (Bartel Solar Rotation 2068 to 2081). The data from Rotation 2060 to 2067 which were used to make forecasts for Rotation 2068 are not shown.

It can be seen from Figs. 2(a) and 2(b) that the levels of geomagnetic activities are quite different for the three zones with relatively subdued activity in the subauroral zone and the most intense activity in the auroral zone. The recurrent high geomagnetic activity, which is mainly due to the long-lived coronal holes co-rotating with the sun, is evident and is punctuated at places by sporadic activity, a good example of which is the intense activities in Rotation 2073. The sporadic activities are related to non-recurrent short-lived solar events such as solar flares and/or filament disappearance. As such, they are unpredictable by the prediction filter which utilizes the recurrence features of the input series. It can be seen from Figs. 2(a) and 2(b) that, except for the very active days due to sporadic activity in the sun, the forecasts generally reproduced the trends of the activities. While one cannot expect one to one correspondence between the forecast values and the actual values, the recurrent peaks evident in the actual data are evident in the forecasts, as can be clearly seen in the first few Bartels rotations in Fig. 2(a).

Obviously, the deviation of the forecast values from the observed values determines the degree of success of the forecast. Figure 3 is a plot of the forecast values vs the actual data for all the data from Rotation 2068 to 2081. If there were no deviation between the forecast values and the observed values, the data points in the plot would fall along the straight line. A noticeable tendency for the values to cluster along the straight line can be seen. The moderately high value of 0.65 for the correlation coefficient between the forecast and data implies a strong linear trend in the scatter diagram.

Another way to evaluate the deviation of the forecast values from the observed values is to construct a histogram of the difference between the two as shown in Fig. 4. It can be seen that about $40 \%$ of the observations lie between the $-10 \mathrm{nT}$ and $+10 \mathrm{nT}$ 


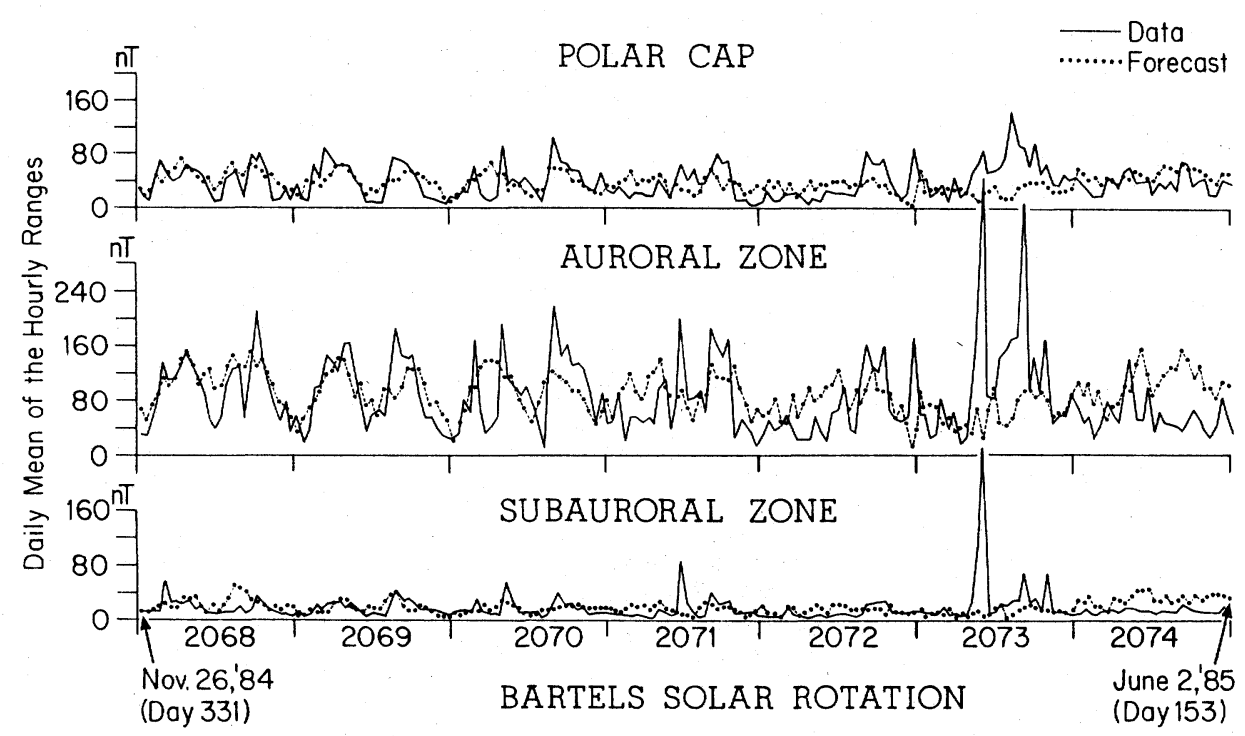

(a)

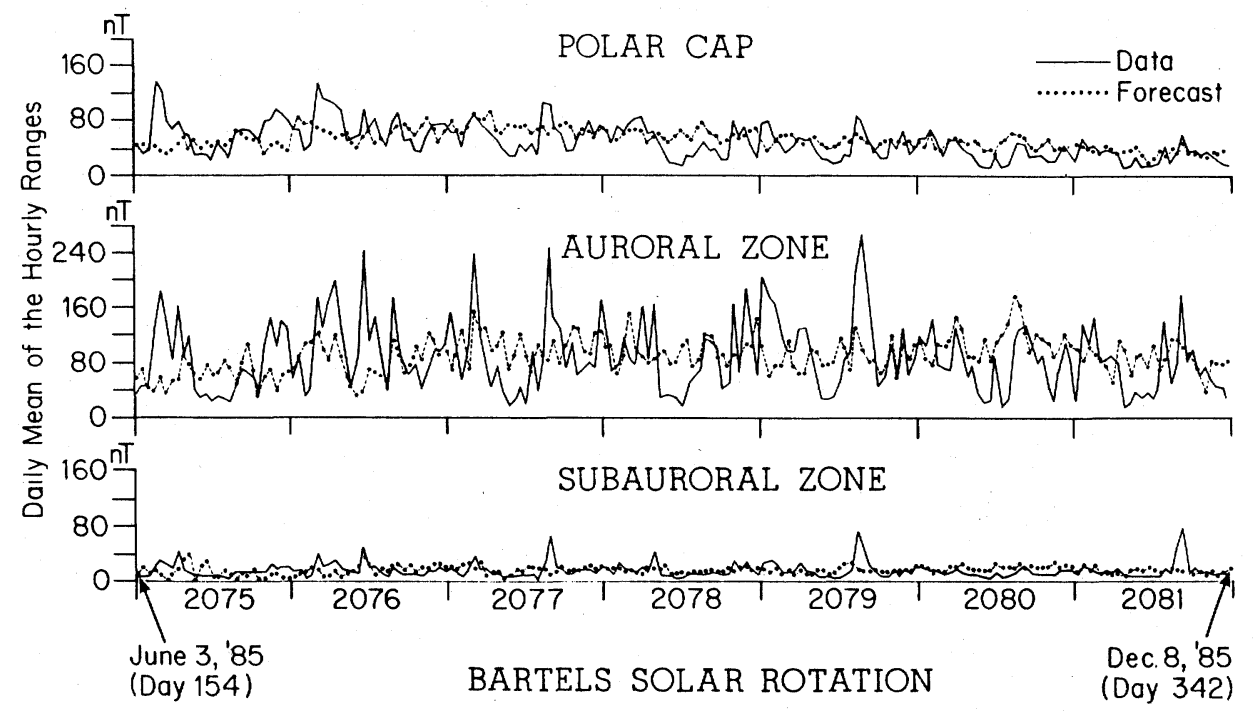

(b)

Fig. 2. (a) Forecast values (dotted line) and actual data (solid line) for the three zones from Bartels Rotation 2068 to 2074 (from November 26, 1984 to June 2, 1985). (b) Same as Fig. 2(a) except for Rotation 2075 to 2081 (from June 3, 1985 to December 8, 1985). 


\section{Rotation 2068 to 2081 \\ Correlation Coefficient $=0.65$}

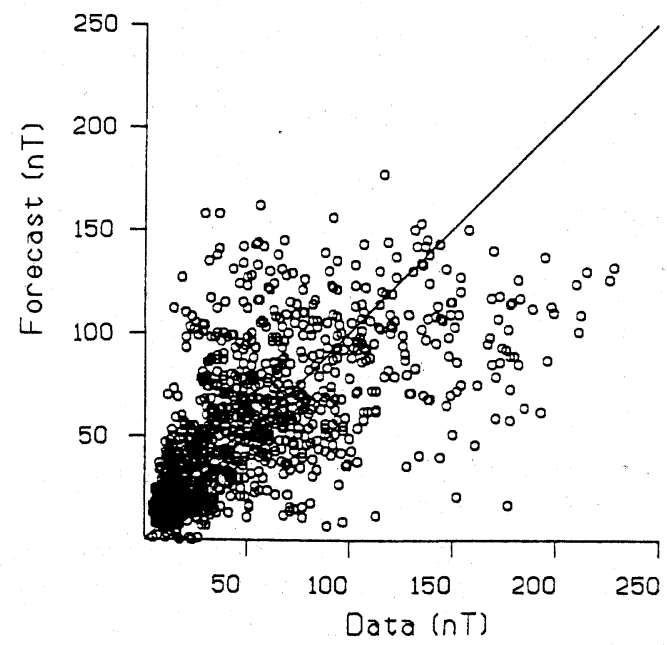

Fig. 3. Forecast values vs actual data for all the data from Rotation 2068 to 2081 . The straight line represents perfect matching between forecast values and observed values.

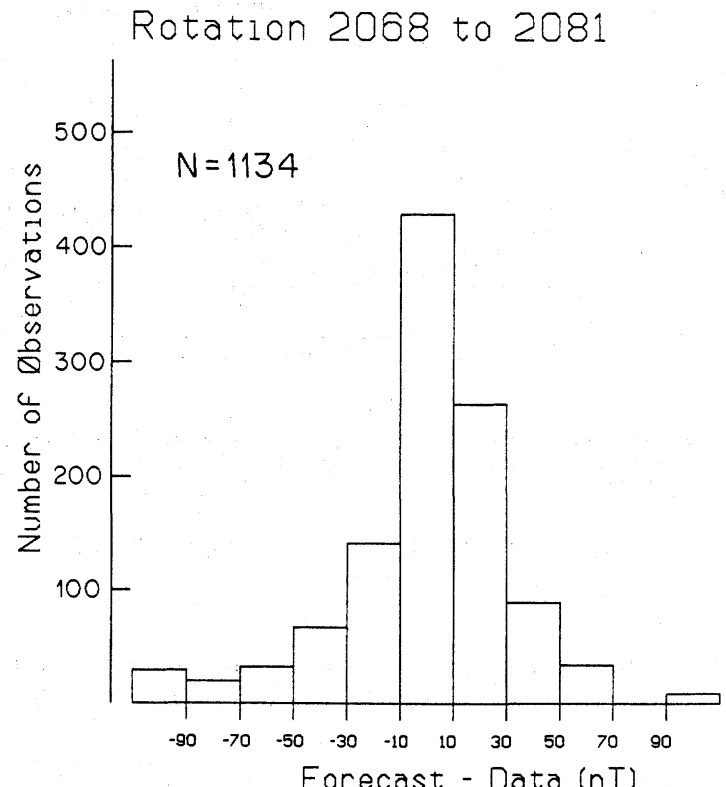

Fig. 4. Histogram of difference between forecast values and actual data. 
range and $75 \%$ of the observations are in the $-30 \mathrm{nT}$ and $+30 \mathrm{nT}$ range. These results show the close agreement between the observed and forecast values.

It is of interest to compare the computer produced forecasts as presented here with the manual forecasts presented by HRUSKA and COLES (1987). Using their definitions of mean absolute deviation $M$ and root mean square deviation $R$, which are both measures of deviation of forecasts from observed values, the values of $M$ for the polar cap, auroral zone and subauroral zone are $19 \mathrm{nT}, 44 \mathrm{nT}$ and $11 \mathrm{nT}$ respectively and those of $R$ are $25 \mathrm{nT}, 59 \mathrm{nT}$ and $19 \mathrm{nT}$ respectively. From HRUSKA and COLES (1987), their values of $M$ for the three zones are $16 \mathrm{nT}, 37 \mathrm{nT}$ and $9 \mathrm{nT}$ and those of $R$ are $28 \mathrm{nT}, 56 \mathrm{nT}$ and $15 \mathrm{nT}$. Although the data set used by Hruska and Coles from the same observatories as utilized here is slightly longer, the values of $M$ and $R$ for both cases are quite comparable. This indicates that the computer forecasts are comparable to the manual forecasts, providing further confidence in using the forecasts by the linear prediction filtering scheme presented here.

\section{Conclusion}

A numerical method of forecasting based on the Wiener linear prediction theory has been tested on about one and a half years of magnetic data from the Canadian Magnetic Observatories. While the filter cannot predict sporadic magnetic activities, the recurrent trends of magnetic activities are well forecast. The filter is therefore particularly useful in times of minimum solar activity when the recurrent tendency is most pronounced. The quantitative forecast values produced by this objective method should serve as an useful aid to a forecaster and reduce the reliance on manual forecasting which can be subjective and at times biased. It has been recently advocated by HRUSKA and COLES $(1984,1987)$ that because of the different latitudinal magnetic characters in Canada as recognized earlier by WHITHAM et al. (1960), the present one zone qualitative geomagnetic forecast for Canada should be replaced by a new three-zone (i.e. polar cap, auroral and subauroral zones) quantitative forecast. The linear prediction technique presented here has been implemented and is now used routinely in the new three-zone forecasts for Canada.

The author wishes to thank Dr. R. L. Coles and Dr. J. Hruska for discussions.

\section{REFERENCES}

HRUSKA, J., Forecasts of geomagnetic activity by Ottawa magnetic observatory: their reliability and application, in Solar-Terrestrial Prediction Proceedings, edited by R. F. Donnelly, Vol. 1, pp. 398-405, U.S. Department of Commerce, Boulder, 1979.

Hruska, J. and R. L. Coles, Recent developments in geomagnetic activity forecasting in Canada, Proc. Workshop Solar-Terrestrial Prediction, Meudon, Paris, June, 1984.

Hruska, J. and R. L. Coles, A new type of magnetic activity forecast for high geomagnetic latitudes, $J$. Geomag. Geoelectr., this issue, 521-534, 1987.

Jansen van Beek, G., R. L. Coles, and L. R. Newitt, Annual Report for Magnetic Observatories 1984, Geomagnetic Series Number 30, 57 pp., Ottawa, Canada, 1986. 
LEvinson, N., The Weiner RMS (root-mean-square) error criterion in filter design and prediction, Appendix in N. Wiener, in Extrapolation, Interpolation and Smoothing of Stationary Time Series with Engineering Applications, pp. 129-148, Tech. Press of the M. I. T. and John Wiley and Sons, Inc., New York, 1949.

Robinson, E. A. and S. Treitel, Principles of digital Wiener filtering, Geophys. Prosp., 15, 311-333, 1967.

Whitham, K., E. I. LOOMER, and E. R. NibletT, The latitudinal distribution of magnetic activity in Canada, J. Geophys. Res., 65, 3961-3974, 1960.

WIENER, N., Extrapolation, Interpolation and Smoothing of Stationary Time Series with Engineering Applications, 163 pp., Tech. Press of the M. I. T. and John Wiley and Sons, Inc., New York, 1949. 